\title{
Transduodenal drainage of symptomatic walled-off pancreatic necrosis in a patient with ansa pancreatica anatomic variation
}

Mateusz Jagielski, Marian Smoczyński, Beata Drelich-Góreczna, Krystian Adrych

Department of Gastroenterology and Hepatology, Medical University of Gdansk, Gdansk, Poland

Submitted: 31 January 2015

Accepted: 5 March 2015

Arch Med Sci 2017; 13, 1: 267-269

DOI: 10.5114/aoms.2017.64724

Copyright @2016 Termedia \& Banach

Endoscopic transmural drainage is an effective treatment for patients with walled-off pancreatic necrosis (WOPN) [1]. Transmural endoscopic drainage of WOPN is based on total removal of the necrotic content through a stoma formed between the lumen of the gastrointestinal tract (stomach or duodenum) and the cavity of the necrotic collection [2].

In 1996 Baron et al. were the first to introduce the results of effective endoscopic treatment of 11 patients with WOPN [3]. After performing gastrocystostomy or duodenocystostomy in the patients, a $10 \mathrm{Fr}$ endoprosthesis and $7 \mathrm{Fr}$ nasocystic drain were inserted transmurally into the cavity of the necrosis in order to irrigate the WOPN [3]. In 2002 a similar method of treatment of patients with pancreatic necrosis was described by Park et al. [4]. Papachristou et al. described transmural drainage of WOPN in 53 patients, with a success rate of $45 / 54(81 \%)$ and the total number of complications 11/53 (21\%) [5]. Seifert et al. introduced 93 patients with WOPN who underwent endoscopic treatment with a success rate of $80 \%$ and complications in $26 \%$ of patients [6]. Smoczyński et al. described the results of endoscopic drainage of WOPN in 112 patients [7]. Long-term success in treatment during 2 years observation was noted in $90.4 \%$ of patients with an average of 2.66 (1-6) endoscopic procedures and average time of active drainage of 25 (4-112) days [7]. Complications were observed in $25.9 \%$ of patients [7].

The direct comparison of the study results is difficult not only because of the different number and variety of patients in study groups, but also because of the differences between treatment methods and aggressiveness of therapy used in the reports cited above.

In the literature, many anatomic variations and congenital disorders of the pancreas are described [8,9]. Most of them have no clinical significance and are detected accidentally in imaging studies $[8,9]$. The loop of the main pancreatic duct (ansa pancreatica) is a very rare anatomic variation that predisposes alcohol-abusing people to acute pancreatitis $[10,11]$.

We have presented a description of the endoscopic treatment of a patient with symptomatic WOPN, who was diagnosed with the ansa pancreatica anatomic variation.

A 65-year-old patient experienced an episode of acute necrotizing pancreatitis of alcoholic etiology in December 2013. The patient was admitted to the Department of Gastroenterology and Hepatology of

\author{
Corresponding author: \\ Mateusz Jagielski MD \\ Department \\ of Gastroenterology \\ and Hepatology \\ Medical University of Gdansk \\ 17 Smoluchowskiego St \\ 80-214 Gdansk, Poland \\ Phone: +48 583493640 \\ Fax: +48 583493650 \\ E-mail: jagiel@gumed.edu.pl
}



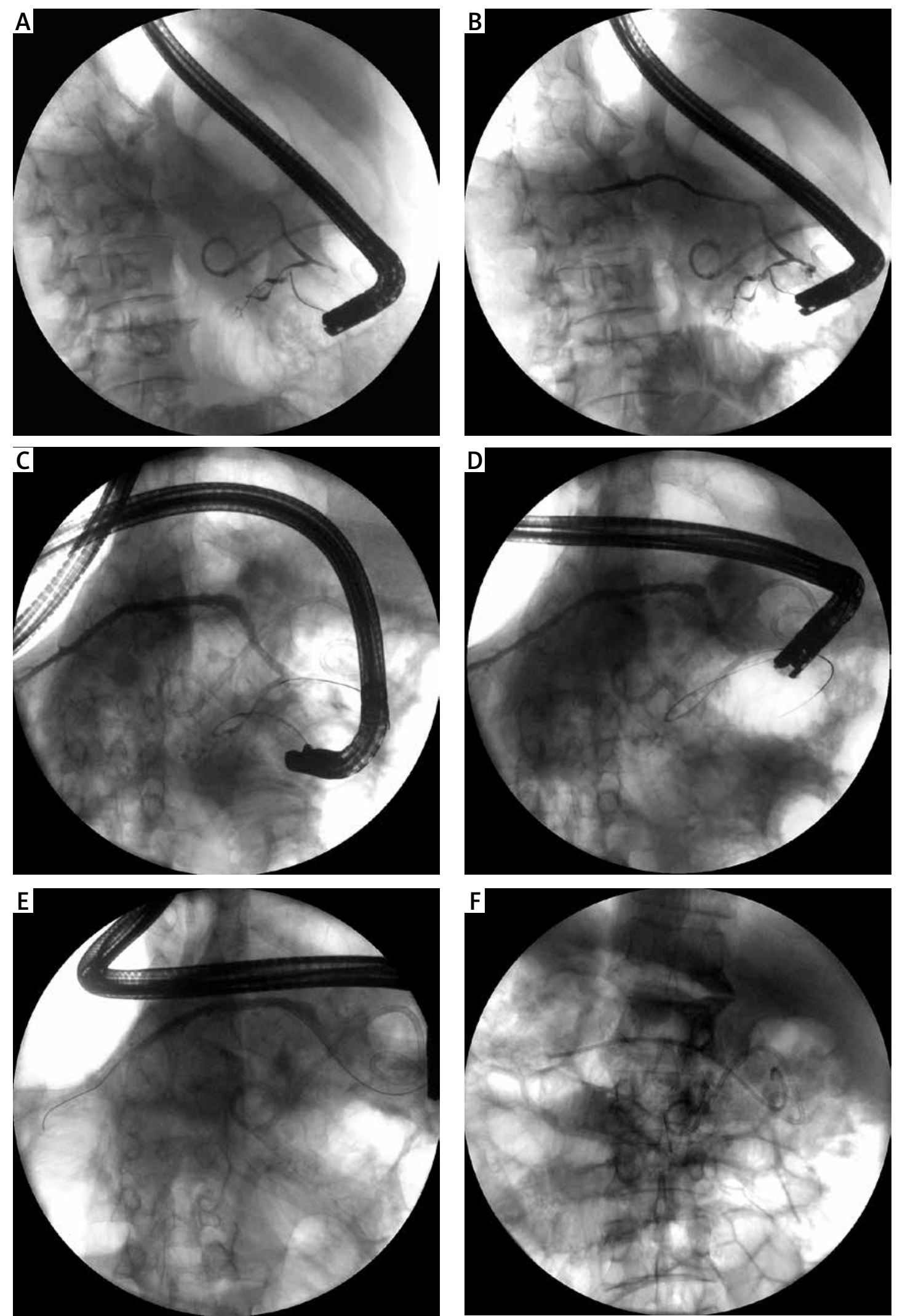

Figure 1. A, B - A contrast medium was introduced through the major duodenal papilla and filled the main pancreatic duct that forms a loop in the head part of the pancreas. Transmurally introduced endoprosthesis is also visible. C, D - Rendezvous maneuver. Through the loop of the pancreatic duct in the head of the pancreas, a guidewire passed out to the lumen of the duodenum through the minor duodenal papilla. E-A guidewire was introduced into the main pancreatic duct through the minor duodenal papilla. F-A pancreatic endoprosthesis was introduced into the main pancreatic duct through the minor duodenal papilla 
Medical University of Gdansk in March 2014, because of abdominal pain and fever up to $38^{\circ} \mathrm{C}$. Contrast-enhanced computed tomography (CECT) of the abdomen was performed and revealed a reservoir of walled-off necrosis in the pancreas head and body of $85 \mathrm{~mm} \times 78 \mathrm{~mm}$ size. During the stay at the clinic an endoscopic transduodenal drainage was performed. Qualification for endoscopic drainage was made on the basis of clinical symptoms related to the presence of fluid collection and contrast-enhanced computed tomography. The place of fistulotomy was chosen under endoscopic ultrasonography (EUS)-guidance where the distance between the collection wall and the gastrointestinal tract wall in EUS did not exceed $1 \mathrm{~cm}$. Enterostomy was performed with a Giovannini cystostome (Cystotome CST-10, Wilson-Cook, Ireland). The stoma between the lumen of the gastrointestinal tract and the cavity of the necrotic collection was widened using an $8 \mathrm{~mm}$ high-pressure balloon (Boston Scientific, USA). Through the stoma an 8.5 Fr nasocystic drain (Wilson-Cook, Ireland) and "double-pigtail" 8 Fr stents (Wilson-Cook, Ireland) were inserted into the cavity of the collection. The necrotic collection was irrigated with saline solution $(200 \mathrm{ml})$ through a nasocystic drain every $2 \mathrm{~h}$ during the first $48 \mathrm{~h}$ and every $4 \mathrm{~h}$ in the subsequent days. After 7 days of active drainage, endoscopic pancreatography was performed to assess the morphology of the main pancreatic duct. The proximal section of the pancreatic duct was cannulated. A contrast medium was introduced through the major duodenal papilla and showed the main pancreatic duct that formed a loop in the head part of the pancreas (Figures $1 \mathrm{~A}, \mathrm{~B}$ ). Ansa pancreatica anatomic variation was diagnosed. Attempts to introduce a guidewire (G-240-3527S, Olympus, Japan) into the duct in the body of the pancreas failed. Moving along the previously described loop in the head of the pancreas, the guidewire passed through the accessory pancreatic duct (duct of Santorini) and through the minor duodenal papilla to the duodenum's lumen (Figures 1 C, D). $A$ rendezvous maneuver was performed. Rattooth forceps (FG-44NR-1, Olympus, Japan) were used to catch the end of the guidewire, which was protruding through the duodenal papilla into the lumen of the duodenum, and then it was guided out through the working channel of the duodenoscope. The other end of the guidewire was left in the lumen of the accessory pancreatic duct. Then, the guidewire was introduced through the duct of Santorini to the distal part of the duct in the body and the tail of the pancreas (Figure $1 \mathrm{E}$ ). Through the minor duodenal papilla a $7 \mathrm{Fr}$ (length $12 \mathrm{~cm}$ ) pancreatic endoprosthesis was introduced into the main pancreatic duct (Figure $1 \mathrm{~F}$ ). The distal end of the endoprosthesis was left in the tail of the pancreas. Because of the absence of symptoms and total regression of the reservoir of the necrosis in the imaging studies, after 14 days of active drainage it was decided to remove the nasogastric tube, leaving the transmural prosthesis only. The patient, without any symptoms, in good general condition, was discharged from the clinic. During the 6-month observation the patient was asymptomatic. A follow-up CECT of the abdomen showed complete resolution of the necrotic reservoir.

\section{Conflict of interest}

The authors declare no conflict of interest.

\section{References}

1. Freeman ML, Werner J, van Santvoort HC, et al. Interventions for necrotizing pancreatitis: summary of a multidisciplinary consensus conference. Pancreas 2012; 41: 1176-94.

2. Baron TH, Kozarek RA. Endotherapy for organized pancreatic necrosis: perspectives after 20 years. Clin Gastroenterol Hepatol 2012; 10: 1202-7.

3. Baron TH, Thaggard WG, Morgan DE, Stanley RJ. Endoscopic therapy for organized pancreatic necrosis. Gastroenterology 1996; 111: 755-64.

4. Park JJ, Kim SS, Koo YS, et al. Definitive treatment of pancreatic abscess by endoscopic transmural drainage. Gastrointest Endosc 2002; 55: 256-62.

5. Papachristou GI, Takahashi N, Chahal P, et al. Peroral endoscopic drainage/debridement of walled-off pancreatic necrosis. Ann Surg 2007; 245: 943-51.

6. Seifert H, Biermer M, Schmitt W, et al. Transluminal endoscopic necrosectomy after acute pancreatitis: a multicentre study with long-term follow-up (the GEPARD Study). Gut 2009; 58: 1260-6.

7. Smoczyński M, Marek I, Dubowik M, et al. Endoscopic drainage/debridement of walled-off pancreatic necrosis - single center experience of 112 cases. Pancreatology 2014; 14: 137-42.

8. Türkvatan A, Erden A, Türkoğlu MA, Yener Ö. Congenital variants and anomalies of the pancreas and pancreatic duct: imaging by magnetic resonance cholangiopancreaticography and multidetector computed tomography. Korean J Radiol 2013; 14: 905-13.

9. Mortelé KJ, Rocha TC, Streeter JL, Taylor AJ. Multimodality imaging of pancreatic and biliary congenital anomalies. Radiographics 2006; 26: 715-31.

10. Tamaka T, Ichiba Y, Miura Y, et al. Variations of the pancreatic ducts as a cause of chronic alcoholic pancreatitis; ansa pancreatica. Am J Gastroenterol 1992; 87: 806.

11. Tamaka T, Ichiba Y, Miura Y, et al. Pathogenesis of chronic alcoholic pancreatitis. Am J Gastroenterol 1990; 85: 1536-7. 